\title{
Plasma cortisol, urinary I7-hydroxycorticoids, and urinary vanilyl mandelic acid after acute myocardial infarction
}

\author{
M. P. Chopra, ${ }^{1}$ U. Thadani, ${ }^{2}$ Clive P. Aber, R. W. Portal, and J. Parkes \\ From the Department of Cardiology, Kingston General Hospital, Hull; \\ and Hull Royal Infirmary
}

Serial estimations of plasma cortisol were made on 24 patients with acute myocardial infarction. Raised levels were found in 23 patients. The maximal level was observed within 12 hours of infarction in 78 per cent and between 12 and 24 hours in 22 per cent of patients. A positive correlation was found between the maximal cortisol level and the maximal levels of serum aspartate aminotransferase and $\alpha$-hydroxybutyrate dehydrogenase.

There was no significant difference between the maximal level in 15 patients with an uncomplicated course and 5 patients who developed primary ventricular fibrillation. The maximal level was significantly higher in 4 patients who died with pump failure.

There was no clear relation between a changing plasma cortisol level and the occurrence of ventricular arrhythmias.

The duration of the cortisol rise varied from II hours to 7 days, and was unrelated to the clinical course or to the maximal cortisol or serum enzyme levels.

No correlation was found between the maximal plasma cortisol level and the initial 24-hour urinary 17-hydroxycorticoids or vanilyl mandelic acid excretion.

Pain, tissue necrosis, and acute circulatory disturbances may provoke adrenocortical and medullary responses. After acute myocardial infarction raised plasma cortisol (Oka, 1956; Klein and Palmer, 1963; Logan and Murdoch, 1966; Bailey, Abernethy, and Beaven, 1967; Jacobs and Nabarro, I969) and high plasma catecholamine levels (Gazes, Richardson, and Woods, 1959; McDonald et al., 1969) have been observed. Furthermore, Jewitt et al. (1969) found an increased urinary catecholamine excretion, which not only appeared to reflect the extent of the myocardial necrosis but was also related to the presence of pulmonary oedema and cardiogenic shock. In their study, the highest urinary catecholamines were recorded in those patients who died. High plasma catecholamine levels have also been associated with an increased incidence of dangerous ventricular arrhythmias after acute myocardial infarction (Gazes et al., 1959; McDonald et al., 1969).

Received ro January 1972.

${ }^{1}$ In receipt of a research grant from Leeds Regional Hospital Board.

2 Present address: Cardiovascular Unit, Leeds General Infirmary.
In the present study we have attempted to relate adrenocortical and medullary activity to the clinical course of acute myocardial infarction and especially to the occurrence of major arrhythmias.

\section{Patients and methods}

Twenty-four patients (22 men and 2 women) with acute myocardial infarction were studied. The age range was 40 to 72 years. All were admitted to a coronary care unit (Aber, Portal, and Chopra, 1969) within 12 hours of infarction. The diagnosis was based on a characteristic clinical history and conventional electrocardiographic criteria (World Health Organization, 1959). All patients had significantly raised serum aspartate aminotransferase (SGOT) and $\alpha$-hydroxybutyrate dehydrogenase (SHBD) levels.

Anticoagulant therapy was given to 16 patients (continuous intravenous heparin 40,000 units daily for 48 hours and warfarin orally). Patients over 65 and those with hypertension, dyspepsia, or haemorrhagic diseases were excluded.

The electrocardiogram of each patient was monitored continuously while in the unit. Arrhythmias were recorded directly by a Miniwriter Mk. II from a master oscilloscope (Cardiac Recorders). Major arrhythmias were defined as 
TABLE Clinical and biochemical data

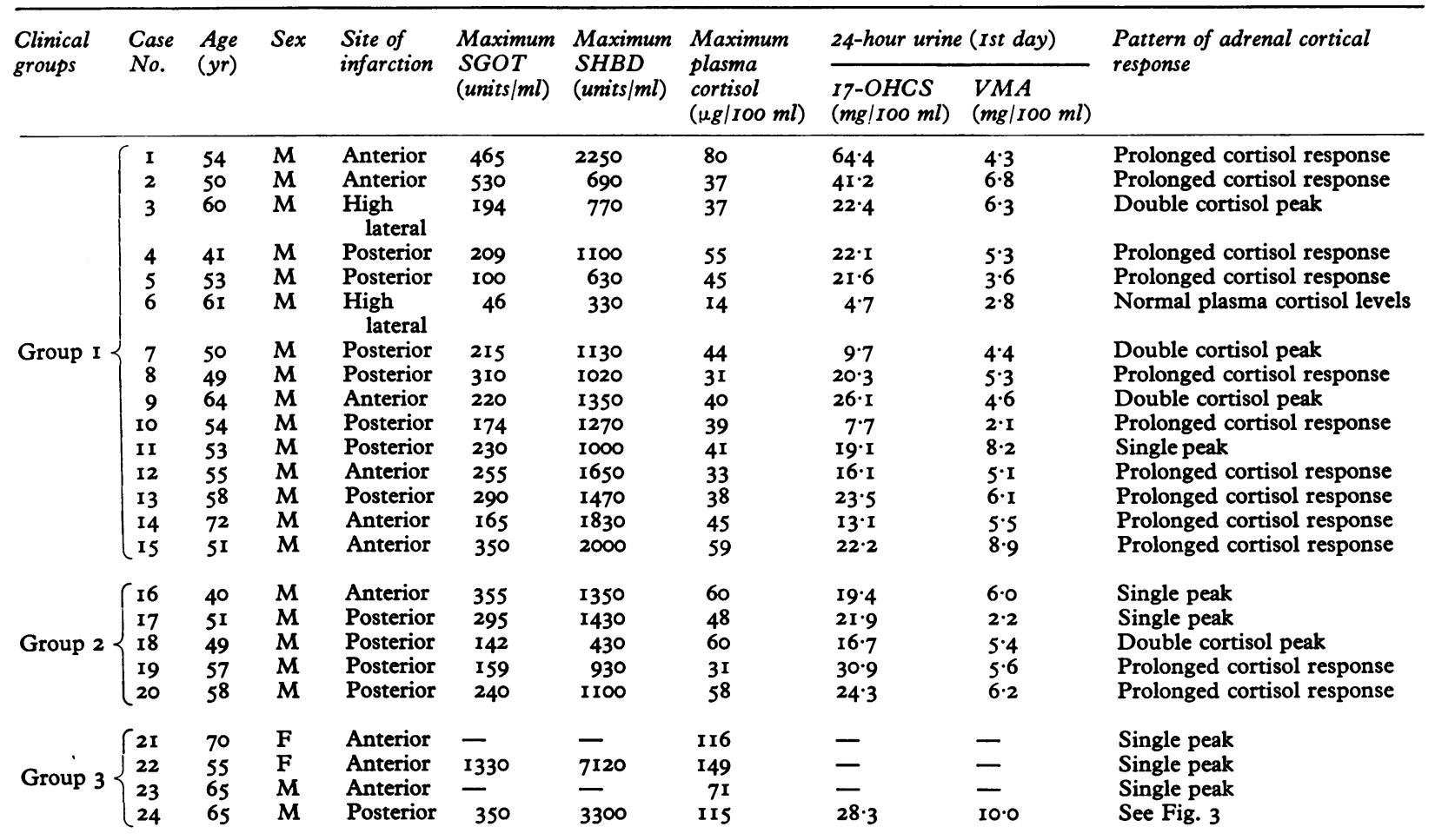

ventricular tachycardia or fibrillation and complete heart block (with or without Adams-Stokes syncope).

The 24 patients have been divided into three clinical groups (Table).

Group I: Uncomplicated (I5 patients) These patients did not develop major arrhythmias, hypotension, circulatory shock, or pulmonary oedema. Two patients showed transient atrial fibrillation on the 2nd and 3rd day after infarction, and 2 others developed frequent unifocal ventricular ectopic activity.

Group 2: Ventricular fibrillation (5 patients) All of these patients experienced cardiac arrest due to primary ventricular fibrillation, but after successful resuscitation 4 had an uncomplicated course. The remaining patient developed frequent unifocal ventricular ectopic activity immediately after successful resuscitation.

Group 3: Death (4 patients) Two of these patients were in circulatory shock and one was in pulmonary oedema on admission. The other patient developed pulmonary oedema and complete heart block with Adams-Stokes syncope after further myocardial infarction on the 14th day. All 4 patients died in ventricular asystole.

Plasma cortisol estimation Plasma cortisol was measured by a modified method of Mattingly (1962). A series of blood samples was collected through a 24-inch polythene catheter (Bardic Deseret Intracath No. 1914 R) introduced into an antecubital vein, so avoiding multiple venepunctures. All specimens were stored at $4^{\circ} \mathrm{C}$ and were estimated within 48 hours of collection. Samples were withdrawn as follows: (a) immediately after admission (within 6 hours of infarction in 17 patients and between 6 and 12 hours in the remaining 7), then half-hourly for the subsequent 3 hours ( 5 specimens) and 4-hourly for the remainder of the 24 hours ( 5 specimens); (b) at 6.0 a.m. on the $3 \mathrm{rd}, 7 \mathrm{th}$, and $14^{\text {th }}$ day after infarction; and (c) in the event of a major arrhythmia or obvious clinical deterioration during the course of the study (e.g. a fall of systolic blood pressure below $90 \mathrm{mmHg}$; the development of pulmonary oedema) further half-hourly samples were taken over a 2-hour period.

Urinary vanilyl mandelic acid and 17hydroxycorticoids estimation Urinary vanilyl mandelic acid (VMA) (Pisano, Crout, and Abraham, 1962) and 17-hydroxycorticoid (17OHCS) (Few, 1961) were measured on 24-hour urine collections on the Ist and I4th days after infarction.

Control group Six male patients aged between 23 and 58 years, without apparent cardiovascular disease and awaiting minor surgical procedures, were used as a control group. Eleven serial plasma cortisol estimations were made on each patient over a 24-hour period, using the same sampling 
technique and time schedule as for the patients with infarcts. Twenty-four hour urine collections were also made for the measurements of vanilyl mandelic acid (VMA) and 17-hydroxycorticoids (17-OHCS) content. Estimations were not made on the 14 th day.

Other measurements a) The heart rate (measured from the electrocardiogram) and the systemic blood pressure were recorded at the time of each blood sampling for plasma cortisol determinations.

b) Estimations of SGOT and SHBD were made on the first 3 days after admission. The SGOT was measured by a modification of the method of Babson et al. (1962) (upper limit of normal 44 Reitman-Frankel units) and SHBD by the spectrophotometric method of Elliott and Wilkinson (I96I) (normal range II4 to 300 Wroblewski units).

c) Arterial blood gas and $\mathrm{pH}$ measurements were made on 12 patients within 12 hours of infarction. Blood samples were taken from the brachial artery and analysed immediately by an Instrumentation Laboratory $p \mathrm{H}$ Blood Gas Analyser (Model II3-SI).

\section{Results}

Plasma cortisol The maximal levels of plasma cortisol in the control group and in the three groups of patients with myocardial infarction are shown in Fig. I. With one exception, all patients with myocardial infarction had raised plasma cortisol levels. Eighteen $(78 \%)$ of these 23 patients showed maximal values within 12 hours, whereas in $5(22 \%)$ this was observed 12 to 24 hours after infarction. The maximal plasma cortisol levels (mean $\pm S D)$ in Group $I(4 \mathrm{I} \cdot 8 \mu \mathrm{g} \pm \mathrm{I4} \cdot 8 \mu \mathrm{g})$ and

FIG. I Maximal plasma cortisol levels after acute myocardial infarction - Control group, Group I (uncomplicated), Group 2 (ventricular fibrillation), and Group 3 (death).

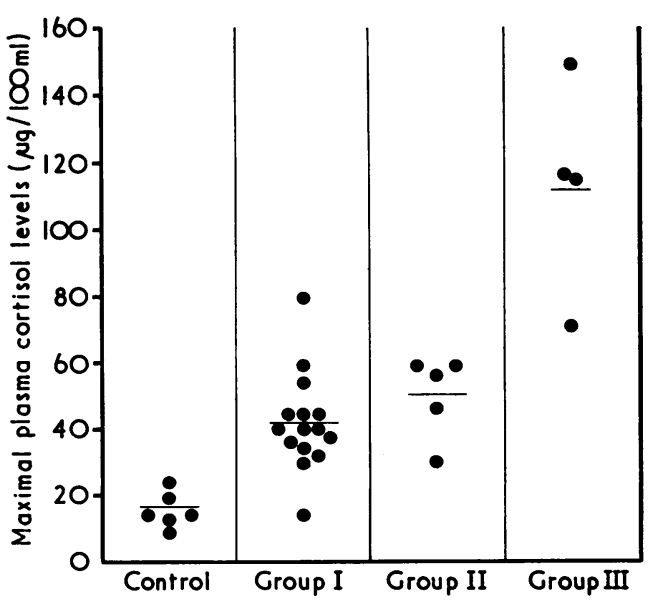

Group $2(5 \mathrm{I} \cdot 4 \mu \mathrm{g} \pm \mathrm{II} \cdot 8 \mu \mathrm{g})$ were significantly higher than in the control group ( $17.2 \mu \mathrm{g} \pm 5.4$ $\mu \mathrm{g})-\mathrm{P}<0.02$. The mean level in Group 2 was not significantly different from that in Group I $(\mathbf{P}>0.7)$. There was a highly significant difference between the maximal plasma cortisol levels in Group 3 (112.7 $\mathrm{g} \pm 32 \mu \mathrm{g})$ and Groups I and 2 ( $P<0.0001)$.

A significant linear correlation $(P<0.0 I)$ was noted between the maximal plasma cortisol levels and the maximal serum enzyme levels.

In Group 2 the maximal plasma cortisol levels were observed immediately after successful resuscitation from primary ventricular fibrillation in 4 patients. A raised plasma cortisol level $(40 \mu \mathrm{g} / 100 \mathrm{ml})$ was also observed after resuscitation in the fifth patient, though the maximal level $(60 \mu \mathrm{g} / 100 \mathrm{ml})$ occurred $\mathrm{I} 4$ hours later as a second peak (Fig. 2) in association with unifocal ventricular ectopic activity. Two other patients showed frequent unifocal ectopic activity coincidental with their maximal plasma cortisol levels.

The highest plasma cortisol levels were observed in patients in Group 3 in association with severe metabolic acidosis, but no constant relation was noted between the maximal plasma cortisol levels and the arterial oxygen tension.

Patterns of adrenal response The expected circadian rhythm was observed in the 6 control patients. After myocardial infarction this rhythm disappeared and on analysis of the postinfarction plasma cortisol values, several facts emerged.

FIG. 2 A double peak plasma cortisol curve after acute myocardial infarction in a patient who experienced recurrent primary ventricular fibrillation and subsequent ventricular ectopic activity (Case 18 in the Table).

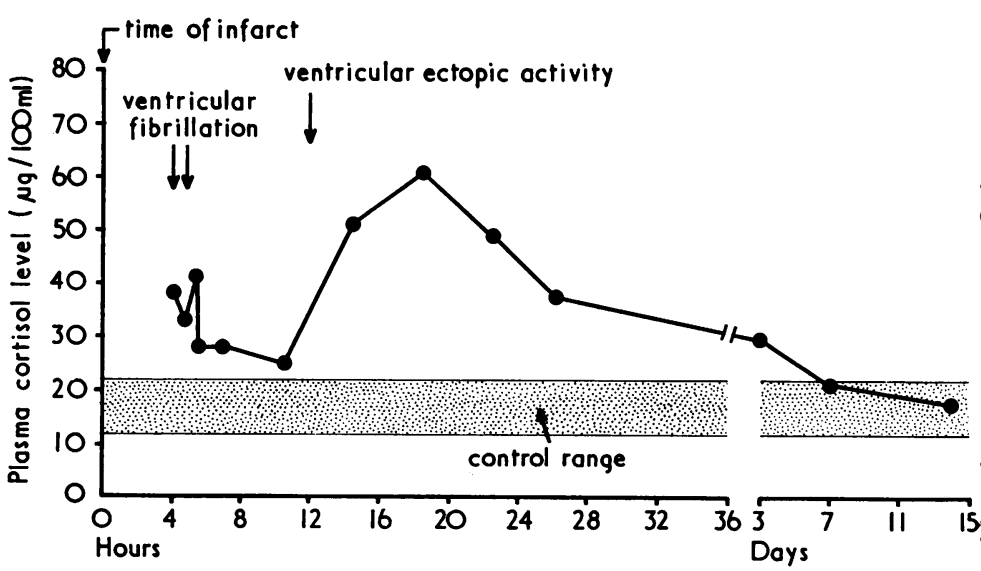


i) The cortisol levels returned to normal values in all the patients who survived. In 3 ( $I$ in Group $I$ and 2 in Group 2) normal values were found within 12 hours. In 12 (10 in Group $I$ and 2 in Group 2) the rise was prolonged from 21 hours to 7 days (Curve A, Fig. 3). The duration of the plasma cortisol increase was unrelated to the maximal cortisol level, the maximal level of serum enzymes, the presence of initial sinus bradycardia (heart rate less than 60 a minute), or the occurrence of frequent ventricular ectopic activity.

ii) Four patients showed a double peak cortisol curve within 24 hours of infarction ( 3 in Group I and I in Group 2). The first peak was observed 2 to 9 hours after infarction. The second peak was seen between 8 and 24 hours after infarction (Curve B, Fig. 3). In I patient the second peak developed at the time of unifocal ventricular ectopic activity (Fig. 2). In 3 it was unrelated to any obvious clinical event.

iii) One patient experienced a second myocardial infarction with acute pulmonary oedema on the 14th day and showed a further substantial rise of the plasma cortisol levels (Curve C, Fig. 3).

Neither the maximal plasma cortisol level nor the duration of cortisol increase appeared to relate to the use of heparin.

Urinary 17-OHCS (21 patients) The 24hour urinary $17-\mathrm{OHCS}$ excretion on the first day after myocardial infarction was within the control range ( $14.3 \mathrm{mg} \pm 3$ ) in 3 patients ( 2 in Group $I$ and $I$ in Group 2) and low in 3 patients (Group I). Increased excretion occurred in 15 patients ( $71 \%$ ) (10 in Group I, 4 in Group 2 and $I$ in Group 3); 9 of these showed a prolonged cortisol rise and 2 a double peak. There was no correlation between the 24-hour urinary 17-OHCS excretion and the maximal plasma cortisol level. The urinary $17-\mathrm{OHCS}$ had returned to the control range or below within I4 days of infarction in all patients except one (Fig. 4).

Urinary VMA (2I patients) The 24-hour urinary excretion of VMA on the first day after myocardial infarction was within the control range $(5.6 \mathrm{mg} \pm \mathrm{I}$ ) or below in $17 \mathrm{pa}$ tients $(81 \%)-12$ in Group $I$ and 5 in Group 2. Four patients ( 3 in Group $I$ ) and $I$ in Group 3) showed high VMA excretion. Two of them had persistent sinus tachycardia and one was in pulmonary oedema. There was an overall significant fall of VMA levels $(P<0.02)$

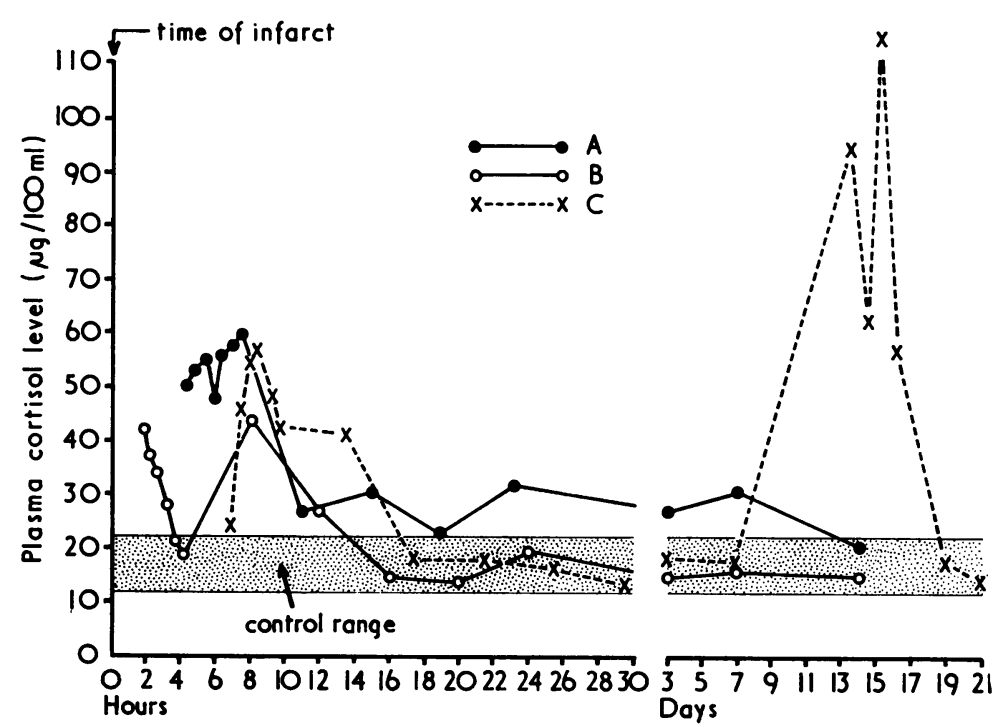

FIG. 3 Plasma cortisol curves after acute myocardial infarction showing $(A)$ a prolonged rise in Case $I,(B)$ a double peak response in Case 7, and $(C)$ a further rise after second infarction on the 14th day in Case 24.

FIG. 4 24-hour urinary excretion of 17hydroxycorticoids on the Ist and 14th days after acute myocardial infarction in $2 I$ patients.

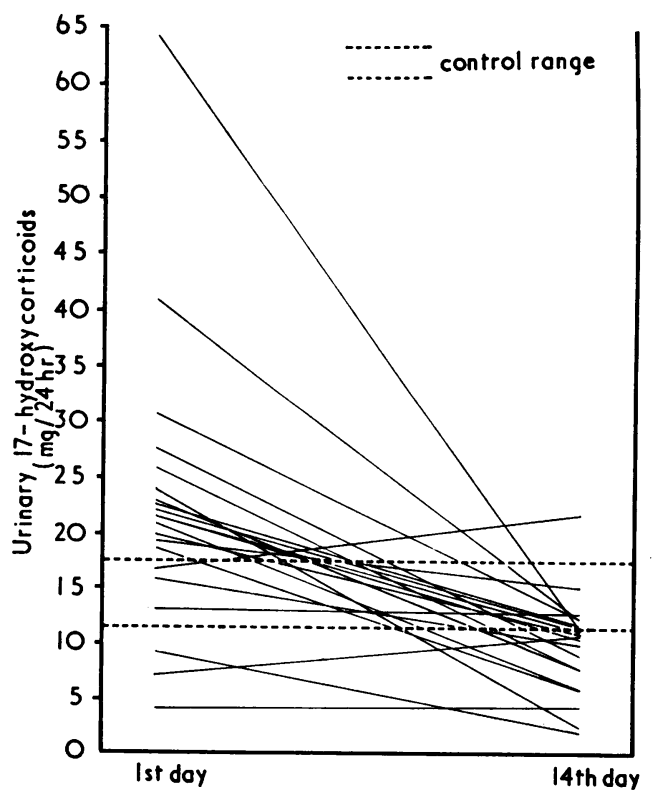


by the fourteenth day. No relation was found between the maximal plasma cortisol level and the urinary VMA excretion.

\section{Discussion}

The results of the present investigation confirm previous reports that the plasma cortisol level is almost invariably raised after myocardial infarction (Oka, I956; Klein and Palmer, I963; Logan and Murdoch, 1966; Bailey et al., 1967) and that the diurnal variation of plasma cortisol secretion is lost under these circumstances (Logan and Murdoch, 1966). Similar adrenocortical responses have been found in association with acute infections, acute psychological stress, and metabolic disturbances (Perkoff et al., 1954; Klein et al., 1955; Murray, 1967; Jacobs and Nabarro, 1969; Levi, 1969).

The maximal plasma cortisol level was usually found within 12 hours and almost always occurred within the first 24 hours of infarction. A clear distinction emerged between the maximal levels in patients with an uncomplicated clinical course and those who subsequently died with pump failure preceded by pulmonary oedema, severe anoxia, and metabolic acidosis. This finding and the observed positive correlation between the maximal cortisol levels and the maximal enzyme levels suggest that the magnitude of the adrenal response reflects the extent of myocardial damage. It is, therefore, not surprising that patients with primary ventricular fibrillation, in whom pump failure had not been evident up to the time of their arrest, showed similar maximal plasma cortisol levels to those with an uncomplicated course. In contrast to the findings of Klein and Palmer (1963), however, the current results show that patients who develop plasma cortisol levels over 40 $\mu \mathrm{g} / \mathrm{roO} \mathrm{ml}$ do not necessarily have a bad prognosis.

The significance of the double peak in the cortisol curve, as seen in 4 patients, is uncertain. Though the second peak coincided with the development of ventricular ectopic activity in I patient and ventricular ectopics were also observed when the plasma cortisol level was maximal in 2 other patients, the present study provides no certain evidence of any causal relation between the cortisol level and the occurrence of ventricular arrhythmia.

Although raised plasma cortisol levels may persist for up to 7 days, the duration of the adrenocortical response did not appear to correlate with any particular pattern of clinical events, but was usually paralleled by an increase in the first 24 -hour urinary $17-\mathrm{OHCS}$ excretion. Whereas a low 17-OHCS urinary excretion level after acute infarction may indicate adrenal exhaustion (Ceremuzynski $e t$ al., 1970), this seems unlikely since the 3 patients with low initial values and the 9 patients with subnormal excretions on the fourteenth day all survived without receiving corticosteroid therapy and none had a low plasma cortisol level. Furthermore, I patient showed a second substantial rapid rise of plasma cortisol (Fig. 3) after sustaining a further myocardial infarction on the fourteenth day. Even so, a beneficial effect from massive doses of corticosteroids has been claimed in cardiogenic shock (Alexander and Azzam, 1969). This, however, has been ascribed to induced vasodilatation and does not imply pre-existing adrenal exhaustion (Lillehei et al., 1964).

The failure to find any obvious relation between the urinary VMA excretion and either the plasma cortisol response or the clinical progress of the patients in this study, is perhaps only to be expected since this measurement is now known to be only a crude index of adrenomedullary activity (Ceremuzynski $e t$ al., 1970).

\section{References}

Aber, C. P., Portal, R. W., and Chopra, M. P. (1969). Cardiac monitoring in a regional hospital. British Medical fournal, $\mathbf{1}, 209$.

Alexander, S., and Azzam, F. G. (1969). Treatment of cardiogenic shock. Medical Clinics of North America, 53, 309.

Babson, A. L., Shapiro, P. O., Williams, P. A. R., and Phillips, G. E. (1962). The use of a diazonium salt for the determination of glutamic-oxalacetic transaminase in serum. Clinica Chimica Acta, 7, 199.

Bailey, R. R., Abernethy, M. H., and Beaven, D. W. (1967). Adrenocortical response to the stress of an acute myocardial infarction. Lancet, $\mathbf{I}, 970$.

Ceremuzynski, L., Kuch, J., Markiewicz, L., Lawecki, J., and Taton, J. (1970). Patterns of endocrine reactivity in patients with recent myocardial infarction. British Heart fournal, 32, 603.

Elliott, B. A., and Wilkinson, J. H. (196I). Serum $\alpha$ hydroxybutyric dehydrogenase in myocardial infarction and in liver disease. Lancet, $1,698$.

Few, J. D. (196I). A method for the analysis of urinary 17-hydroxy-corticosteroids. Fournal of Endocrinology, 22, 31.

Gazes, P. C., Richardson, J. A., and Woods, E. F. (1959). Plasma catechol amine concentrations in myocardial infarction and angina pectoris. Circulation, 19, 657.

Jacobs, H. S., and Nabarro, J. D. N. (1969). Plasma II-hydroxycorticosteroid and growth hormone levels in acute medical illnesses. British Medical fournal, 2, 595.

Jewitt, D. E., Mercer, C. J., Reid, D., Valori, C., Thomas, M., and Shillingford, J. P. (1969). Free noradrenaline and adrenaline excretion in relation to the development of cardiac arrhythmias and heart-failure in patients with acute myocardial infarction. Lancet, $1,635$. 
Klein, A. J., and Palmer, L. A. (1963). Plasma cortisol in myocardial infarction - a correlation with shock and survival. American fournal of Cardiology, II, 332.

Klein, R., Papadatos, C., Fortunato, J., and Byers, C. (I955). Acid-hydrolyzable corticoids of serum. fournal of Clinical Endocrinology and Metabolism, 15, 215.

Levi, L. (1969). Neuro-endocrinology of anxiety. In Studies of Anxiety (British fournal of Psychiatry, Special Publication No. 3), p. 40. Ed. by M. H. Lader. Headley, London.

Lillehei, R. C., Longerbeam, J. K., Bloch, J. H., and Manax, W. G. (1964). The nature of irreversible shock: experimental and clinical observations. Annals of Surgery, 160, 682.

Logan, R. W., and Murdoch, W. R. (1966). Bloodlevels of hydrocortisone, transaminases, and cholesterol after myocardial infarction. Lancet, 2, 521 .

McDonald, L., Baker, C., Bray, C., McDonald, A., and Restieaux, N. (I969). Plasma-catecholamines after cardiac infarction. Lancet, 2, 102 I.

Mattingly, D. (1962). A simple fluorimetric method for the estimation of free II-hydroxycorticoids in human plasma. fournal of Clinical Pathology, 15, 374.

Murray, D. (I967). Cortisol binding to plasma proteins in man in health, stress and at death. fournal of Endocrinology, 39, 571.

Oka, M. (1956). Effects of myocardial infarction and stenocardia on the level of 17-hydroxycorticosteroids in plasma. Acta Medica Scandinavica, 156, 227.

Perkoff, G. T., Sandberg, A. A., Nelson, D. H., and Tyler, F. H. (1954). Clinical usefulness of determination of circulating 17-hydroxycorticosteroid levels. Archives of Internal Medicine, 93, I.

Pisano, J. J., Crout, J. R., and Abraham, D. (1962). Determination of 3-methoxy-4-hydroxymandelic acid in urine. Clinica Chimica Acta, 7, 285.

World Health Organization (1959). Hypertension and coronary heart disease: classification and criteria for epidemiological studies. First report of the expert committee on cardiovascular diseases and hypertension. Technical Report Series, No. I68.

Requests for reprints to Dr. Clive P. Aber, The Cardiac Department, Kingston General Hospital, Hull, HU3 rUR. 